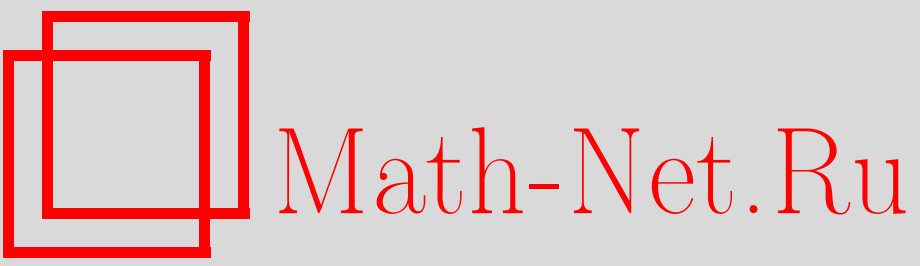

И. А. Шакиров, Об уточнении асимптотической формулы для функции Лебега полинома Лагранжа, Итоги науки и техн. Сер. Соврем. мат. и ее прил. Tемат. обз., 2021, том 192, 142-149

DOI: https://doi.org/10.36535/0233-6723-2021-192-142-149

Использование Общероссийского математического портала Math-Net.Ru подразумевает, что вы прочитали и согласны с пользовательским соглашением

http://www.mathnet.ru/rus/agreement

Параметры загрузки:

IP: 3.82 .47 .9

26 апреля 2023 г., 05:40:14 


\title{
ОБ УТОЧНЕНИИ АСИМПТОТИЧЕСКОЙ ФОРМУЛЫ ДЛЯ ФУНКЦИИ ЛЕБЕГА ПОЛИНОМА ЛАГРАНЖА
}

\author{
(c) 2021 г. $\quad$ И. А. ШАКИРОВ
}

\begin{abstract}
АннотАция. Для функции Лебега, соответствующей интерполяционному полиному Лагранжа,
\end{abstract} получены строгие оценки снизу и сверху и уточнение известной асимптотической формулы.

Ключевъе слова: полином Лагранжа, функция Лебега, асимптотическая формула, равномерная оценка остаточного члена.

\section{ON THE REFINEMENT OF THE ASYMPTOTIC FORMULA FOR THE LEBESGUE FUNCTION OF THE LAGRANGE POLYNOMIAL}

\section{(C) 2021 I. A. SHAKIROV}

\begin{abstract}
For the Lebesgue function corresponding to the Lagrange interpolation polynomial, strict lower and upper estimates are obtained and the well-known asymptotic formula is refined.

Keywords and phrases: Lagrange polynomial, Lebesgue function, asymptotic formula, uniform remainder estimate.
\end{abstract}

AMS Subject Classification: $34 \mathrm{~A} 25$

1. Введение. При изучении поточечной сходимости общеизвестного интерполяционного полинома Лагранжа

$$
\Phi_{n}^{*}(x, t)=\frac{1}{n} \sum_{k=1}^{2 n} D_{n}^{*}\left(t_{k}-t\right), \quad D_{n}^{*}(t)=\frac{\sin n t}{2 \operatorname{tg}(t / 2)}, \quad t_{k}=\frac{\pi k}{n}, \quad n \in \mathbb{N}
$$

к исходной функции $x(t) \in C_{2 \pi}$, принципиальное значение имеет поведение функции Лебега

$$
\lambda_{n}^{*}(t)=\frac{1}{n} \sum_{k=1}^{2 n}\left|D_{n}^{*}\left(t_{k}-t\right)\right|, \quad t \in T^{*}=[0, \pi / n],
$$

где $D_{n}^{*}(u)$ - видоизмененное ядро Дирихле, $C_{2 \pi}=\{x(t) \mid x(t) \in C[0,2 \pi], x(0)=x(2 \pi)\}, T^{*}-$ основной период функции (2), $n$ - степень полинома $(1)$.

В математической литературе для функции (2) известна асимптотическая формула

$$
\lambda_{n}^{*}(t)=\frac{2}{\pi} \ln n \sin n t+O(1), \quad n \rightarrow+\infty .
$$

В ее правой части содержится неопределенная ограниченная величина $O(1)$, которая появляется в связи с издержками метода получения формулы (3) из (2). Действительно, в процессе исключения модулей в сумме (2) и ее последовательном преобразовании при больших значениях параметра $n(n \gg 1)$ появляются промежуточные неопределенные ограниченные величины 
$O_{k}(1)\left(O(1)=\sum_{k} O_{k}(1)\right)$. Они являются следствием (продуктом) замены сложных функций и конечных сумм более простыми агрегатами при выделении главной составляющей а правой части формулы (3). Контролировать допущенную погрешность на каждом этапе не представляется возможным. Сведения об этих и других проблемах, возникающих при интерполировании функций, можно найти в монографиях [1-6].

Таким образом, до сих пор остается неясным поведение предельной величины $O(1)$. При конечных значениях параметра $n$ аналогичный вопрос является не менее актуальным и для остаточного члена $O_{n}(t)$ в представлении функции Лебега вида

$$
\lambda_{n}^{*}(t)=\frac{2}{\pi} \ln n \sin n t+O_{n}(t), \quad t \in T^{*}, \quad O_{n}(t) \equiv \lambda_{n}^{*}(t)-\frac{2}{\pi} \ln n \sin n t, \quad n \in \mathbb{N} .
$$

Ниже убедимся, что изучение поведения остаточных членов, входящих в формулы (3) и (4), будет более продуктивным, если при этом использовать явное выражение функции Лебега (см. [7])

$$
\lambda_{n}^{*}(t)=\frac{1}{2 n}\left[\sum_{k=1}^{n}\left(\operatorname{ctg} \frac{t_{k-1}+t}{2}+\operatorname{ctg} \frac{t_{k}-t}{2}\right)\right] \sin n t, \quad t \in T^{*}, \quad t_{k}=\frac{\pi k}{n}, \quad n \in \mathbb{N} .
$$

В рамках данной работы последовательно решены следующие новые задачи:

(1) при помощи явного выражения (5) функции Лебега получены строгие оценки снизу и сверху логарифмо-тригонометрическими полиномами (формула (16));

(2) затем с помощью этих оценок уточнено поведение остаточного члена $O_{n}(t)$, который с малой погрешностью представлен в виде $O_{n}(t) \approx a_{n} \sin n t+b_{n}, n \in \mathbb{N}$, где коэффициенты $a_{n}=a(n)$, $b_{n}=b(n)$ - вполне определенные функциональные зависимости (см. (19)-(21));

(3) получена более общая, чем (3), асимптотическая формула

$$
\lambda_{n}^{*}(t)=\frac{2}{\pi} \ln n \cdot \sin n t+a \cdot \sin n t+b, \quad n \rightarrow+\infty,
$$

где

$$
a=\frac{1}{2}-\frac{1}{2 \pi}-\frac{1}{\pi} \ln \frac{\pi}{2}=0,197101817 \ldots, \quad b=\frac{1}{2}+\frac{1}{\pi}=0,818309886 \ldots ;
$$

(4) входящие в формулы (3), (4) остаточные члены равномерно оценены сверху вполне определенной константой.

Ясно, что асимптотическая формула (6) является обобщением и уточнением формулы (3). Ниже увидим, что возможность представления $\lambda_{n}^{*}(t)$ в таком виде напрямую связана с преимуществами явного представления (5) этой функции (см. [7]) перед формулой (2). Различные явные виды функций Лебега, соответствующие тригонометрическим интерполяционным полиномам Лагранжа, впервые введены и подробно исследованы автором в [7-9].

2. Вспомогательные сведения. Нам понадобятся классы функций $V_{\delta}^{+}$и $V_{\delta}^{-}$.

Определение 1. Строго монотонная функция $\varphi=\varphi(n), n \in D=D(\varphi) \subseteq \mathbb{N}$ дискретного аргумента принадлежит одному из классов $V_{\delta}^{ \pm}$, если для изменения (вариации)

$$
\delta_{\varphi} \equiv \delta(\varphi)=\sup \{\varphi(n) \mid n \in D\}-\inf \{\varphi(n) \mid n \in D\} \quad\left(\delta_{\varphi}>0\right)
$$

области ее значения $R(\varphi)$ выполняется условие $\delta_{\varphi}<\delta$, где $\delta$-заранее выбранное положительное число. В обозначении класса используется знак плюс $\left(V_{\delta}^{+}\right)$в случае возрастания функций в области $D$, знак минус $\left(V_{\delta}^{-}\right)$- при их убывании.

Ясно, что эти классы определены как семейство функций $\{\varphi\}$, для вариации каждого из которых выполняется неравенство $\delta_{\varphi}<\delta$. Участвующие в леммах и теоремах функции $\varphi$ в основном имеют малые изменения областей их значений $R(\varphi)$, следовательно, и малые вариации $\delta_{\varphi}\left(\delta_{\varphi} \ll 1\right)$. Даже для самой «худшей» из них $\hat{\varphi}=\hat{\varphi}(n), n \in D$ в нашем случае имеет место неравенство $\delta(\hat{\varphi})<0,138$. 
Замечание 1. Функции (последовательности) из классов $V_{\delta}^{+}$и $V_{\delta}^{-}$обладают тем замечательным свойством, что наибольшие их вариации происходят при первоначальных значениях аргумента $n(n=1 \vee n=\overline{1,2} \vee n=\overline{1,3})$ с последующей их «стабилизацией» около вполне определенных предельных точек. Данное свойство с успехом используется для получения более тонких (сильных) оценок в утверждениях работы.

Ниже применим непрерывные продолжения дискретных функций $\varphi=\varphi(n), n \in D \subset \mathbb{N}$ из классов $V_{\delta}^{ \pm}$на области $\bar{D}=\bar{D}(\varphi) \subset \mathbb{R}$. Они позволят использовать методы дифференциального исчисления для доказательства некоторых вспомогательных лемм работы.

Замечание 2. Для непрерывных продолжений $\bar{\varphi}=\bar{\varphi}(n), n \in \bar{D}(\bar{D}=\overline{\inf D ; \sup D} \subset \mathbb{R})$ дискретно определенных функций $\varphi=\varphi(n)(n \in D \subset \mathbb{N})$ формулировка и суть определения 1 полностью сохраняются.

Для простоты формулировок, а также с целью исключения из рассмотрения тривиального случая $(n=1)$ далее всюду положим значение параметра принадлежащим области $D^{*}=\{n \mid$ $n \geqslant 2\} \subset \mathbb{N}$.

Приведем необходимые в дальнейшем две леммы и две теоремы из работ автора [10,11].

Лемма 1. Функиия дискретного аргумента

$$
\alpha_{n}^{*} \equiv \alpha^{*}(n)=1 /[n \sin (\pi / 2 n)], \quad n \in D^{*} \quad\left(n=1 \Rightarrow \alpha_{1}^{*}=1\right)
$$

является строго убывающей функиией; для ее образа и вариации верны соотношения

$$
R\left(\alpha_{n}^{*}\right)=(2 / \pi, \sqrt{2} / 2] \subset(0,636 ; 0,708) ; \quad \delta=\delta\left(\alpha_{n}^{*}\right)=\sqrt{2} / 2-2 / \pi=0,072 \ldots,
$$

т.е. она принадлежит классу $V_{\delta}^{-}$.

Лемма 2. Функииональные зависимости

$$
\begin{aligned}
& \varphi_{1}^{*} \equiv \varphi_{1}^{*}(n)=1-\alpha_{n}^{*} \quad\left(R\left(\varphi_{1}^{*}\right)=[1-\sqrt{2} / 2,1-2 / \pi) \subset(0,292,0,364), \quad \delta\left(\varphi_{1}^{*}\right)=0,072 \ldots\right), \\
& \varphi_{2}^{*} \equiv \varphi_{2}^{*}(n)=\alpha_{n}^{*} \cos (\pi /(2 n)) \quad\left(R\left(\varphi_{2}^{*}\right)=[1 / 2,2 / \pi) \subset(0,499,0,637), \quad \delta\left(\varphi_{2}^{*}\right)=0,137 \ldots\right),
\end{aligned}
$$

определенные в области $D^{*}$ и выраженные через (7), являются функииями из класса $V_{\delta}^{+}$.

Теорема 1. Функиия Лебега $\lambda_{n}^{*}(t), t \in T^{*}\left(n \in D^{*}\right)$, соответствующая интерполяционному полиному (1),

(i) является $\pi / n$-периодической, четной функцией;

(ii) на полупериоде $T_{0}^{*}=[0, \pi / 2 n] \subset T^{*}$ строго возрастает, а в его дополнении строго убывает; выпукла вверх всюду в области определения;

(iii) в области $T_{0}^{*}$ представима в виде

$$
\lambda_{n}^{*}(t)=l_{n}^{*}(t)+a_{n}^{*}(t) \sin n t \quad\left(n \in D^{*} ; \quad n=1 \Rightarrow \lambda_{1}^{*}(t) \equiv 1\right),
$$

əде

$$
l_{n}^{*}(t) \stackrel{\text { def }}{=} \frac{\sin n t}{n \sin t}, \quad a_{n}^{*}(t) \stackrel{\text { def }}{=} \frac{1}{2 n} \sum_{k=1}^{n-1}\left(\operatorname{ctg} \frac{t_{k}-t}{2}+\operatorname{ctg} \frac{t_{k}+t}{2}\right) \quad\left(n=1 \Rightarrow l_{1}^{*}(t) \equiv 1, \quad a_{1}^{*}(t) \equiv 0\right) .
$$

При вышеуказанных значениях аргумента $t$ и параметра $n$ не только функции (10), но и сама функция Лебега (9) принимают только положительные значения, т.е. $l_{n}^{*}(t)>0, a_{n}^{*}(t)>0$, $\lambda_{n}^{*}(t)>0, t \in T_{0}^{*}\left(n \in D^{*}\right)$.

Четность $\lambda_{n}^{*}(t)$ позволяет сформулировать следующую теорему лишь для полупериода $T_{0}^{*}$.

Теорема 2. Для функиий из (10) в области $T_{0}^{*}$ справедливы следующие утверждения:

(i) $l_{n}^{*}(t)$ является выпукло убывающей функиией, и для нее верна двусторонняя оценка

$$
\alpha_{n}^{*} \leqslant l_{n}^{*}(t) \leqslant 1, \quad t \in T_{0}^{*}, \quad l_{n}^{*}(0) \stackrel{\text { def }}{=} \lim _{t \rightarrow 0+} l_{n}^{*}(t)=1, \quad l_{n}^{*}(\pi /(2 n))=\alpha_{n}^{*}, \quad n \in D^{*} ;
$$

(ii) $a_{n}^{*}(t)$ является строго возрастающей, выпуклой вниз функиией, для которой имеет место двойное неравенство

$$
a_{n}^{*}(0) \leqslant a_{n}^{*}(t) \leqslant a_{n}^{*}(\pi / 2 n), \quad t \in T_{0}^{*} \quad\left(n \in D^{*}\right) .
$$


3. Основные результаты. Исследование поведения функции Лебега $\lambda_{n}^{*}(t)$ при $n \rightarrow \infty$ и $n \in \mathbb{N}$, установление асимптотических, а также приближенных формул с оценкой допущенной погрешности относятся к актуальным задачам теории приближения функции. Ниже подробно изучены эти и другие вопросы, имеющие непосредственное отношение к формуле (4).

Используя приведенные в предыдущем пункте вспомогательные результаты, вначале установим две леммы, которые позволят доказать основные теоремы и, таким образом, решить поставленные во введении задачи.

Лемма 3. Функииональные зависимости (последовательности)

$$
\begin{aligned}
\varphi_{3}^{*} & \equiv \varphi_{3}^{*}(n)=\frac{1}{2} \varphi_{2}^{*}(n)+\frac{2}{\pi} \ln \alpha_{n}^{*}, \quad n \in D^{*}, \\
\varphi_{4}^{*} & \equiv \varphi_{4}^{*}(n)=\varphi_{1}^{*}(n)-\varphi_{3}^{*}(n), \quad n \in D^{*},
\end{aligned}
$$

выраженные через определенные в леммах 1-3 последовательности, принадлежат классу $V_{\delta}^{+}$, имеют характеристики

$$
\begin{array}{ll}
R\left(\varphi_{3}^{*}\right)=\left[\frac{1}{4}-\frac{1}{\pi} \ln 2, \frac{1}{\pi}-\frac{2}{\pi} \ln \frac{\pi}{2}\right) \subset(0,029 ; 0,031), & \delta\left(\varphi_{3}^{*}\right)=0,001 \ldots \\
R\left(\varphi_{4}^{*}\right)=\left[\frac{3}{4}-\frac{1}{\pi} \ln 2-\frac{\sqrt{2}}{2}, 1+\frac{2}{\pi} \ln \frac{\pi}{2}-\frac{3}{\pi}\right) \subset(0,263 ; 0,333), & \delta\left(\varphi_{4}^{*}\right)=0,069 \ldots
\end{array}
$$

Доказательство. С целью исследования поведения функции (13) непрерывно продолжим ее на область $\bar{D}^{*}=[2,+\infty) \subset \mathbb{R}$ (см. замечание 2$)$. Она представлена в виде суммы двух составляющих и является гладкой функцией. Согласно леммам 2 и 1 первая из них в рассматриваемой области строго возрастает, а вторая - строго убывает (логарифмическая функция сохраняет свойство монотонности).

Сказанное требует более детального исследования функции $\varphi_{3}^{*}(n), n \in D^{*}$ с использованием дифференциальных методов. Предварительно ее несколько преобразуем:

$$
\varphi_{3}^{*}(n)=\frac{1}{2} \varphi_{2}^{*}(n)+\frac{2}{\pi} \ln \alpha_{n}^{*}=\frac{1}{\pi} \cdot \frac{\pi}{2 n} \cdot \frac{\cos (\pi / 2 n)}{\sin (\pi / 2 n)}+\frac{2}{\pi} \ln \frac{\pi / 2 n}{\sin (\pi / 2 n)}+\frac{2}{\pi} \ln \frac{2}{\pi}, \quad n \in \bar{D}^{*} .
$$

Замена переменой $x=\pi / 2 n(n \in[2,+\infty) \Rightarrow x \in(0, \pi / 4])$ позволяет записать полученное представление в виде

$$
\tilde{\varphi}_{3}^{*}(x)=\frac{1}{\pi} x \cdot \operatorname{ctg} x+\frac{2}{\pi} \ln \frac{x}{\sin x}+\frac{2}{\pi} \ln \frac{2}{\pi}, \quad x \in\left(0, \frac{\pi}{4}\right],
$$

а затем определить знак ее производной с использованием разложения Маклорена:

$$
\begin{aligned}
\left(\tilde{\varphi}_{3}^{*}(x)\right)^{\prime} & =\frac{1}{\pi}\left(\operatorname{ctg} x-\frac{x}{\sin ^{2} x}\right)+\frac{2}{\pi} \cdot \frac{\sin x}{x}\left(\sin x-\frac{x \cos x}{\sin ^{2} x}\right)= \\
& =\frac{x}{\pi x \sin ^{2} x}\left(2 \sin ^{2} x-x^{2}-x \sin x \cos x\right)= \\
& =-\frac{x}{\pi x \sin ^{2} x}\left(\frac{2}{45} x^{6}-\frac{2}{315} x^{8}+\ldots\right)<0 \quad \forall x \in\left(0, \frac{\pi}{4}\right] .
\end{aligned}
$$

Поэтому $\tilde{\varphi}_{3}^{*}(x)(x \in(0, \pi / 4])$ является строго убывающей функцией, а исходная функция $\varphi_{3}^{*}(n)$ $(n \in[2,+\infty))$ - строго возрастающей в области своего определения. Следовательно, $\varphi_{3}^{*}(n) \in V_{\delta}^{+}$.

Функцию $\varphi_{4}^{*}(n)$, представленную в виде разности двух функций из класса $V_{\delta}^{+}$, как и в предыдущем случае исследуем с использованием производной. Замена переменой $x=\pi / 2 n(n \in[2,+\infty) \Rightarrow$ $x \in(0, \pi / 4])$ позволяет выразить функцию (14) через новый аргумент в виде

$$
\tilde{\varphi}_{4}^{*}(x)=1-\frac{2}{\pi} \cdot \frac{x}{\sin x}-\frac{1}{\pi} x \operatorname{ctg} x-\frac{2}{\pi} \ln \frac{x}{\sin x}-\frac{2}{\pi} \ln \frac{2}{\pi}, \quad x \in\left(0, \frac{\pi}{4}\right] .
$$


Ее производную изучим с применением разложения в степенной ряд:

$$
\begin{aligned}
\left(\tilde{\varphi}_{4}^{*}(x)\right)^{\prime} & =-\frac{1}{\pi}\left[2 \frac{\sin x-x \cos x}{\sin ^{2} x}+\frac{\sin x \cos x-x}{\sin ^{2} x}+2 \frac{\sin x-x \cos x}{x \sin x}\right]= \\
& =-\frac{1}{\pi x \sin ^{2} x}\left(2 x \sin x-2 x^{2} \cos x-x \sin x \cos x+2 \sin ^{2} x-x^{2}\right)= \\
& =-\frac{1}{\pi x \sin ^{2} x}\left(\frac{2}{3} x^{4}-\frac{1}{9} x^{6}+\ldots\right)<0 \quad \forall x \in\left(0, \frac{\pi}{4}\right] .
\end{aligned}
$$

Повторяя предыдущие рассуждения, имеем: $\varphi_{4}^{*}(n), n \in[2,+\infty)$ является строго возрастающей функцией, т.е. $\varphi_{4}^{*}(n) \in V_{\delta}^{+}$. Приведенные в лемме характеристики функций (13) и (14) устанавливаются без особых усилий. Лемма 3 полностью доказана.

Лемма 4. Для функиии $a_{n}^{*}(t)$ из (10) равномерно относительно ее аргумента верно двустороннее неравенство

$$
\varphi_{3}^{*}(n)+\frac{2}{\pi} \ln n<a_{n}^{*}(t)<\varphi_{1}^{*}(n)+\frac{2}{\pi} \ln n, \quad t \in T_{0}^{*} \quad\left(n \in D^{*}\right),
$$

где последовательности $\left(\varphi_{1}^{*}\right),\left(\varphi_{3}^{*}\right)$ определены в (8) и (13) соответственно.

Доказательство. Если значение параметра $n=1$, то имеем тривиальный случай: $\varphi_{1}^{*}(1)=0$, $\varphi_{3}^{*}(1)=0, a_{1}^{*}(t) \equiv 0$.

Вначале несколько преобразуем $a_{n}^{*}(\pi / 2 n)$, входящую в правую часть (12); затем, используя неулучшаемую верхнюю оценку для константы Лебега вида $\lambda_{n}^{*} \leqslant 1+(2 / \pi) \ln n, n \in \mathbb{N}\left(\lambda_{n}^{*}<\right.$ $\left.1+(2 / \pi) \ln n, n \in D^{*}\right)$ (см. [10,12,13]), оценим ее сверху:

$$
\begin{aligned}
a_{n}^{*}(\pi / 2 n) & =\frac{1}{2 n} \sum_{k=1}^{n-1}\left(\operatorname{ctg} \frac{t_{k}-\pi / 2 n}{2}+\operatorname{ctg} \frac{t_{k}+\pi / 2 n}{2}\right)= \\
& =\frac{1}{2 n}\left[\sum_{k=1}^{n} \operatorname{ctg} \frac{t_{k}-\pi / 2 n}{2}+\sum_{k=0}^{n-1} \operatorname{ctg} \frac{t_{k}+\pi / 2 n}{2}-\operatorname{ctg} \frac{t_{0}+\pi / 2 n}{2}-\operatorname{ctg} \frac{t_{n}-\pi / 2 n}{2}\right]= \\
& =\lambda_{n}^{*}-\frac{1}{2 n}\left[\operatorname{ctg} \frac{\pi}{4 n}-\operatorname{ctg}\left(\frac{\pi}{2}-\frac{\pi}{4 n}\right)\right]= \\
& =\lambda_{n}^{*}-\alpha_{n}^{*}<1-\alpha_{n}^{*}+\frac{2}{\pi} \ln n \equiv \varphi_{1}^{*}(n)+\frac{2}{\pi} \ln n, \quad t_{k}=\frac{\pi}{n} k .
\end{aligned}
$$

Теперь оценим $a_{n}^{*}(0)$ снизу:

$$
\begin{aligned}
a_{n}^{*}(0) & =\frac{1}{2 n} \sum_{k=1}^{n-1}\left(\operatorname{ctg} \frac{t_{k}-0}{2}+\operatorname{ctg} \frac{t_{k}+0}{2}\right)=\frac{1}{n} \sum_{k=1}^{n-1} \operatorname{ctg} \frac{t_{k}}{2}=\frac{1}{\pi} \sum_{k=1}^{n-1}\left(\operatorname{ctg} \frac{t_{k}}{2}\right) \cdot \frac{\pi}{n}> \\
& >\frac{1}{\pi}\left[\int_{t_{1}}^{\pi} \operatorname{ctg}(t / 2) d t+\frac{1}{2} \cdot \frac{\pi}{n} \cdot \operatorname{ctg} \frac{t_{1}}{2}\right]=\frac{1}{\pi}\left[\left.2 \ln \sin \frac{t}{2}\right|_{t_{1}} ^{\pi}+\frac{\pi}{2 n} \cdot \frac{\cos (\pi / 2 n)}{\sin (\pi / 2 n)}\right]= \\
& =\frac{2}{\pi} \ln \frac{n}{n \sin (\pi / 2 n)}+\frac{\cos (\pi / 2 n)}{2} \cdot \frac{1}{n \sin (\pi / 2 n)}=\frac{1}{2} \alpha_{n}^{*} \cos \frac{\pi}{2 n}+\frac{2}{\pi} \ln \alpha_{n}^{*}+\frac{2}{\pi} \ln n \equiv \\
& \equiv \varphi_{3}^{*}(n)+\frac{2}{\pi} \ln n,
\end{aligned}
$$

где $\varphi_{3}^{*}(n)$ определена в (13). Лемма доказана.

Заметим, что среди всевозможных представлений [9] функции Лебега только формула (9) позволяет осуществить строгую двустороннюю ее оценку. Используя эту формулу и результаты предыдущих двух лемм, ниже решим эту задачу. 
Теорема 3. Для функиии Лебега $\lambda_{n}^{*}(t), t \in T_{0}^{*}(n \geqslant 2)$ справедлива следующая двусторонняя оченка:

$$
\frac{2}{\pi} \ln n \cdot \sin n t+\varphi_{3}^{*}(n) \cdot \sin n t+\alpha_{n}^{*}<\lambda_{n}^{*}(t) \leqslant \frac{2}{\pi} \ln n \cdot \sin n t+\varphi_{1}^{*}(n) \cdot \sin n t+1,
$$

где $\alpha_{n}^{*}, \varphi_{1}^{*}, \varphi_{3}^{*}$ ранее изученные последовательности (см. (7), (8), (13)).

Доказательство. Для получения верхней оценки в (16) используем формулу (9), неравенства (11), (12), (15):

$$
\begin{gathered}
\lambda_{n}^{*}(t)=l_{n}^{*}(t)+a_{n}^{*}(t) \sin n t \leqslant \max _{t \in T_{0}^{*}} l_{n}^{*}(t)+\left[\max _{t \in T_{0}^{*}} a_{n}^{*}(t)\right] \cdot \sin n t=l_{n}^{*}(0)+a_{n}^{*}\left(\frac{\pi}{2 n}\right) \sin n t \leqslant \\
\leqslant 1+\left[\varphi_{1}^{*}(n)+\frac{2}{\pi} \ln n\right] \cdot \sin n t=1+\varphi_{1}^{*}(n) \cdot \sin n t+\frac{2}{\pi} \ln n \cdot \sin n t, \quad t \in T_{0}^{*} \quad(n \geqslant 2),
\end{gathered}
$$

где равенства достигаются лишь при значении аргумента $t=0(n \in \mathbb{N})$.

Нижнюю оценку в (16) получим, применяя результаты теоремы 2 и неравенство (15):

$$
\begin{aligned}
\lambda_{n}^{*}(t)= & l_{n}^{*}(t)+a_{n}^{*}(t) \sin n t \geqslant \min _{t \in T_{0}^{*}} l_{n}^{*}(t)+\left[\min _{t \in T_{0}^{*}} a_{n}^{*}(t)\right] \cdot \sin n t=l_{n}^{*}\left(\frac{\pi}{2 n}\right)+a_{n}^{*}(0) \cdot \sin n t> \\
& >\alpha_{n}^{*}+\left[\varphi_{3}^{*}(n)+\frac{2}{\pi} \ln n\right] \cdot \sin n t=\frac{2}{\pi} \ln n \cdot \sin n t+\varphi_{3}^{*}(n) \cdot \sin n t+\alpha_{n}^{*}, \quad t \in T_{0}^{*} \quad(n \geqslant 2) .
\end{aligned}
$$

Теорема доказана.

Замечание 3. Появление нестрогого неравенства в верхней оценке (16) связано с тем, что при значении аргумента $t=0$ из области $T_{0}^{*}$ значение функции Лебега всегда равно единице, т.е. $\lambda_{n}^{*}(0)=1$ для всех $n \in D^{*}$.

Результат теоремы 3 позволяет теперь установить более точную, чем (3), асимптотическую формулу для функции (2).

Теорема 4. Для функиии $\lambda_{n}^{*}(t), t \in T_{0}^{*}$, верно асимптотическое равенство

$$
\lambda_{n}^{*}(t) \cong \frac{2}{\pi} \ln n \cdot \sin n t+a \cdot \sin n t+b, \quad n \rightarrow+\infty,
$$

где

$$
a=\frac{1}{2}-\frac{1}{2 \pi}-\frac{1}{\pi} \ln \frac{\pi}{2}=0,197101817 \ldots, \quad b=\frac{1}{2}+\frac{1}{\pi}=0,818309886 \ldots
$$

Доказательство. Используемая здесь терминология «асимптотическая формула» и символ $\cong$ не гарантируют совпадения предельных значений левой и правой частей в формуле (17); в случае их совпадения имеем «асимптотически точную формулу».

В предыдущей теореме функция (9) была достаточно строго оценена с двух сторон. Найдем полусумму $\eta_{n}^{*}(t)$ верхней и нижней оценивающих $\lambda_{n}^{*}(t)$ функций в неравенстве $(16)$ :

$$
\begin{aligned}
\eta_{n}^{*}(t) \stackrel{\text { def }}{=} \frac{2}{\pi} \ln n \cdot \sin n t+\frac{1}{2}\left[\varphi_{1}^{*}(n)+\varphi_{3}^{*}(n)\right] \sin n t & +\frac{1}{2}\left[1+\alpha_{n}^{*}\right]= \\
& =(2 / \pi) \ln n \cdot \sin n t+a_{n} \cdot \sin n t+b_{n}, \quad n \in D^{*},
\end{aligned}
$$

где

$$
a_{n} \equiv a(n)=\left(\varphi_{1}^{*}+\varphi_{3}^{*}\right) / 2 \in V_{\delta}^{+}, \quad b_{n} \equiv b(n)=\left(1+\alpha_{n}^{*}\right) / 2 \in V_{\delta}^{-} .
$$

Подробные сведения о последовательностях $\alpha_{n}^{*}, \varphi_{1}^{*}(n), \varphi_{3}^{*}(n)$ содержатся в леммах 1-3. Справедливость принадлежностей $a_{n} \in V_{\delta}^{+}$и $b_{n} \in V_{\delta}^{-}$не вызывает сомнения, так как они являются 
линейными комбинациями (с положительными коэффициентами) последовательностей из соответствующих классов. Их характеристики имеют вид

$$
\begin{array}{ll}
R\left(a_{n}\right)=\left[\frac{5}{8}-\frac{\sqrt{2}}{4}-\frac{1}{2 \pi} \ln 2, \frac{1}{2}-\frac{1}{2 \pi}-\frac{1}{\pi} \ln \frac{\pi}{2}\right) \subset(0,161 ; 0,198), & \delta\left(a_{n}\right)=0,035 \ldots, \\
R\left(b_{n}\right)=\left(\frac{1}{2}+\frac{1}{\pi}, \frac{1}{2}+\frac{\sqrt{2}}{4}\right] \subset(0,818 ; 0,854), & \delta\left(b_{n}\right)=0,037 \ldots
\end{array}
$$

Теперь для функции Лебега $\lambda_{n}^{*}(t)$ за приближающий ее агрегат примем функцию (18), т.е.

$$
\lambda_{n}^{*}(t) \approx \eta_{n}^{*}(t), \quad t \in T^{*}, \quad n \in D^{*} \Longleftrightarrow O_{n}(t) \approx a_{n} \sin n t+b_{n},
$$

а за асимптотическую формулу - предельное значение $\eta_{n}^{*}(t)$ при $n \rightarrow+\infty$ функции (18), т.е.

$$
\lambda_{n}^{*}(t) \cong \frac{2}{\pi} \ln n \cdot \sin n t+\left(\lim _{n \rightarrow+\infty} a_{n}\right) \cdot \sin n t+\lim _{n \rightarrow+\infty} b_{n}=\frac{2}{\pi} \ln n \cdot \sin n t+a \cdot \sin n t+b, \quad n \rightarrow+\infty,
$$

где согласно (19) и (20) имеем

$$
a=\lim _{n \rightarrow+\infty} a_{n}=\frac{1}{2}-\frac{1}{2 \pi}-\frac{1}{\pi} \ln \frac{\pi}{2}=0,197101817 \ldots, \quad b=\lim _{n \rightarrow+\infty} b_{n}=\frac{1}{2}+\frac{1}{\pi}=0,818309886 \ldots
$$

Теорема доказана.

Следствие. Если в условиях теоремы 4 значение аргумента $t$ положим равным серединному значению $\pi / 2 n$ периода $T^{*}$ функции Лебега $\left(\lambda_{n}^{*}(\pi / 2 n) \stackrel{\text { def }}{=} \lambda_{n}^{*}\right)$, то асимптотическое представление (17) с учетом (19) и (20) превращается в асимптотическую формулу для соответствующей константы Лебега вида

$$
\lambda_{n}^{*} \cong \frac{2}{\pi} \ln n+\bar{\alpha}_{0}, \quad n \rightarrow+\infty, \quad \bar{\alpha}_{0}=1+\frac{1}{2 \pi}-\frac{1}{\pi} \ln \frac{\pi}{2}=1,015411703 \ldots
$$

Она незначительно отличается от известной (см. [12,13] асимптотически точной формулы

$$
\lambda_{n}^{*}=\frac{2}{\pi} \ln n+\alpha_{0}, \quad n \rightarrow+\infty, \quad \alpha_{0}=\frac{2}{\pi}\left[\gamma+\ln \frac{8}{\pi}\right]=0,962522826 \ldots
$$

для константы $\lambda_{n}^{*}$. Действительно, их правые части различаются на малую величину

$$
\delta \stackrel{\text { def }}{=} \bar{\alpha}_{0}-\alpha_{0}=1-\frac{4 \gamma-1}{2 \pi}-\frac{1}{\pi} \ln \frac{32}{\pi}=0,052888876 \ldots
$$

( $\gamma=0,577215664 \ldots$ - константа Эйлера), что является одним из показателей качества формулы (17).

Примечание. Асимптотическая формула (17) является естественным обобщением (уточнением) общеизвестной формулы (3), в которой $O(1)$ уточнена как

$$
\left(\frac{1}{2}-\frac{1}{2 \pi}-\frac{1}{\pi} \ln \frac{\pi}{2}\right) \sin n t+\frac{1}{2}+\frac{1}{\pi}
$$

С целью дальнейшей равномерной оценки допущенной в приближенном равенстве (21) абсолютной погрешности введем в рассмотрение функцию погрешности

$$
\varepsilon_{n}^{*}(t) \stackrel{\text { def }}{=}\left|\lambda_{n}^{*}(t)-\eta_{n}^{*}(t)\right| \quad\left(\varepsilon_{n}^{*}(t) \geqslant 0 \quad \forall t \in T^{*}, \quad n \in D^{*}\right)
$$

и экстремальную величину

$$
\varepsilon^{*} \stackrel{\text { def }}{=} \sup _{n \geqslant 2} \max _{t \in T^{*}} \varepsilon_{n}^{*}(t)=\sup _{n \geqslant 2} \max _{t \in T^{*}}\left|\lambda_{n}^{*}(t)-\eta_{n}^{*}(t)\right| .
$$

Теорема 5. Для функиии погрешности (22) верна равномерная по переменным $t$ и $n$ оценка

$$
\varepsilon^{*}=\sup _{n \geqslant 2} \max _{t \in T^{*}}\left|\lambda_{n}^{*}(t)-\eta_{n}^{*}(t)\right|<0,348 .
$$


Доказательство. Абсолютное значение погрешности в приближенной формуле (21) всегда меньше либо равно полуразности верхней и нижней оценивающих функций в двойном неравенстве (16), т.е.

$$
\left|\lambda_{n}^{*}(t)-\eta_{n}^{*}(t)\right| \leqslant \frac{1}{2}\left|\left(\varphi_{1}^{*}-\varphi_{3}^{*}\right) \cdot \sin n t+1-\alpha_{n}^{*}\right|=\frac{1}{2}\left(\varphi_{4}^{*} \sin n t+\varphi_{1}^{*}\right),
$$

где свойства синуса, характеристики функций $\varphi_{1}^{*}(n), \varphi_{4}^{*}(n)$, определенные в леммах 1 и 3 , позволили в его правой части исключить модуль из рассмотрения.

Переходя последовательно к максимуму и супремуму в полученном неравенстве, и учитывая при этом характеристики функций $(8),(14)$ из класса $V_{\delta}^{+}$, получим требуемую оценку $(23)$ :

$$
\sup _{n \geqslant 2} \max _{t \in T^{*}}\left|\lambda_{n}^{*}(t)-\eta_{n}^{*}(t)\right| \leqslant \frac{1}{2}\left[\sup _{n \in D^{*}} \varphi_{4}^{*}(n)+\sup _{n \in D^{*}} \varphi_{1}^{*}(n)\right] \Longleftrightarrow \varepsilon^{*} \leqslant 1+\frac{1}{\pi} \ln \frac{\pi}{2}-\frac{5}{2 \pi}
$$

т.е. $\varepsilon^{*}<0,348$. Теорема доказана.

Оценим сверху остаточный член $O_{n}(t)$ и его предельное значение $O(1)$ равномерно относительно $t, n$. Для этого используем результат предыдущей теоремы и проведем несложные расчеты с учетом соотношений (18)-(20):

$$
\begin{aligned}
\left|O_{n}(t)\right|=\mid \lambda_{n}^{*}(t) & -\frac{2}{\pi} \ln n \sin n t|\leqslant| \lambda_{n}^{*}(t)-\frac{2}{\pi} \ln n \sin n t-a_{n} \sin n t-b_{n}|+| a_{n} \sin n t+b_{n} \mid \leqslant \\
\leqslant & \varepsilon_{n}^{*}(t)+\left(a_{n}+b_{n}\right)<\left(1+\frac{1}{\pi} \ln \frac{\pi}{2}-\frac{5}{2 \pi}\right)+\left(1+\frac{1}{2 \pi}-\frac{1}{\pi} \ln \frac{\pi}{2}\right)=2-\frac{2}{\pi}<1,364
\end{aligned}
$$

для всех $t \in T^{*}, n \in \overline{\mathbb{N}}$, где дополнительно использованы свойства последовательности

$$
\varphi_{6}^{*}(n) \equiv a_{n}+b_{n}=1+\frac{1}{2} \varphi_{3}^{*}(n) \in V_{\delta}^{+}, \quad R\left(\varphi_{6}^{*}\right)=\left[\frac{9}{8}-\frac{1}{2 \pi} \ln 2,1+\frac{1}{2 \pi}-\frac{1}{\pi} \ln \frac{\pi}{2}\right) \subset(1,014 ; 1,016) .
$$

\section{СПИСОК ЛИТЕРАТУРЫ}

1. Дзядык В. К. Аппроксимационные методы решения дифференциальных и интегральных уравнений. - Киев: Наукова думка, 1988.

2. Зигмунд А. Тригонометрические ряды. - М.: Мир, 1965.

3. Корнейчук Н. П. Экстремальные задачи теории приближения. - М.: Наука, 1976.

4. Натансон И. П. Конструктивная теория функций. - М.-Л.: Гостехиздат, 1949.

5. Привалов A. А. Теория интерполирования функций. Т. 1, 2. - Саратов: Изд-во Саратов. ун-та, 1990.

6. Тиман $A$. $\Phi$. Теория приближения функций действительного переменного. - М.: Физматгиз, 1960.

7. Шакиров И. А. О тригонометрическом интерполяционном полиноме Лагранжа, имеющем минимальную норму как оператор из в// Изв. вузов. Мат. - 2010. - 10. - С. 60-68.

8. Шакиров И. А. Полное исследование функций Лебега, соответствующих классическим интерполяционным полиномам// Изв. вузов. Мат. - 2011. - 10. - С. 80-88.

9. Шакиров И. А. О функциях Лебега, соответствующих семейству интерполяционных полиномов Лагранжа// Изв. вузов. Мат. - 2013. - 7. - С. 77-89.

10. Шакиров И. А. О влиянии выбора узлов лагранжевой интерполяции на точные и приближенные значения констант Лебега// Сиб. мат. ж. - 2014. - 55, № 6. - С. 1404-1423.

11. Шакиров И. А. Асимптотические формулы для функций Лебега, соответствующих семейству интерполяционных полиномов Лагранжа// Мат. заметки. - 2017. - 102, № 1. - С. 133-147.

12. Brutman L. Lebesgue functions for polynomial interpolation. A survey// Ann. Numer. Math. — 1997. 4. - P. 111-127.

13. Rivlin T. The Lebesgue constants for polynomial interpolation/ Functional Analysis and Its Application. — Berlin: Springer-Verlag, 1974.

Шакиров Искандер Асгатович

Набережночелнинский государственный педагогический университет

E-mail: iskander@tatngpi.ru 